\title{
FATORES RELACIONADOS AO DELIRIUM E À MORTALIDADE EM IDOSOS VÍTIMAS DE FRATURA DE FÊMUR EM UMA ENFERMARIA DE ORTOPEDIA
}

\author{
Factors related to delirium and mortality in \\ older adults with femur fracture on an orthopedic unit
}

\author{
Daniel de Souza Oliveira a,* $\odot$, Fernanda Marques Fernandes ${ }^{\mathrm{a}} \odot$, \\ Mara Graziele Maciel Silveira ${ }^{a} \odot$, Maurício de Miranda Ventura ${ }^{\circledR}$ ๑)
}

\begin{abstract}
OBJETIVO: Correlacionar as principais características clínicas do idoso internado por fratura de fêmur com a incidência de delirium e mortalidade durante a internação. MÉTODOS: Estudo transversal em pacientes com idade acima de 65 anos internados em enfermaria de ortopedia com fratura de fêmur. Foi aplicada aos pacientes e/ou cuidadores uma entrevista elaborada pelos autores para levantamento de suas características clínicas, sendo posteriormente realizada a correlação entre o perfil e a taxa de delirium e mortalidade. Para análise estatística, foi usado o programa Statistical Package for the Social Sciences (SPSS), $o$ teste $t$ de Student e o teste do $\chi^{2}$. Foi considerado estatisticamente significativo o valor $p<0,05$. RESULTADOS: Noventa pacientes com média de idade de 83 anos (65-99), sendo 77,8\% mulheres. O tempo médio de internação foi de 19,7 dias, sendo identificado delirium em 39,9\% dos pacientes, e a taxa de mortalidade foi de 17,8\%. O delirium esteve significativamente associado à idade avançada ( $p=0,046$ ), ao uso de psicotrópicos — especialmente a quetiapina —, à maior taxa de mortalidade, ao diabetes mellitus, à síndrome demencial e à baixa funcionalidade. Mortalidade, por sua vez, apresentou associação com insuficiência renal crônica, menor funcionalidade e síndrome demencial. CONCLUSÃO: Encontramos que o delirium esteve associado a idades mais elevadas, à menor funcionalidade, ao diabetes mellitus, à síndrome demencial, ao maior desfecho de óbitos e ao uso de psicotrópicos; e a mortalidade, à síndrome demencial, à insuficiência renal crônica e à pior funcionalidade.

PALAVRAS-CHAVE: geriatria; fraturas do fêmur; delirium; mortalidade.
\end{abstract}

OBJECTIVE: To correlate the main clinical characteristics of geriatric patients hospitalized for femur fracture with delirium incidence and mortality during hospitalization. METHODS: Cross-sectional study in patients over 65 years old admitted to an orthopedics unit with femoral fracture. The authors interviewed patients and / or caregivers to investigate their clinical characteristics, and subsequently correlate their profiles to the rates of delirium and mortality. Statistical analysis was performed using the Statistical Package for Social Sciences (SPSS), Student's $t$-test and $\chi^{2}$ test. Significance was established at $p<0.05$. RESULTS: Participants included 90 patients with a mean age of 83 years (65-99), 77.8\% women. The mean length of stay was 19.7 days, with delirium identified in $39.9 \%$ of patients, and a mortality rate of $17.8 \%$. Delirium was significantly associated with advanced age $(p=0.046)$, use of psychotropic drugs — especially quetiapine — , higher mortality rate, diabetes mellitus, dementia syndrome, and low functionality. Mortality, in turn, was associated with chronic kidney disease, lower functionality, and dementia syndrome. CONCLUSION: We found that delirium was associated with advanced age, lower functionality, diabetes mellitus, dementia syndrome, higher death outcomes, and the use of psychotropic drugs; and mortality was associated to dementia syndrome, chronic kidney disease, and worse functionality.

KEYWORDS: geriatrics; femoral fractures; delirium; mortality.

aServiço de Geriatria, Instituto de Assistência Médica ao Servidor Público Estadual - São Paulo (SP), Brasil.

Dados para correspondência

Daniel de Souza Oliveira - Rua Doutor Diogo de Faria, 1.104, ap. 102 - Vila Clementino - CEP: 04037-003 - São Paulo (SP), Brasil. E-mail: danieldesouzaoliveira@gmail.com

Recebido em: 11/03/2019. Aceito em: 26/03/2019

DOI: 10.5327/Z2447-211520191900020 


\section{INTRODUÇÃO}

Delirium é uma complicação comum nas internações de idosos, estando também presente de forma significativa nas enfermarias de ortopedia. Esse diagnóstico é associado com diversos desfechos negativos, incluindo declínio cognitivo, dependência, institucionalização e mortalidade. ${ }^{1} \mathrm{O}$ fator de risco mais consistentemente relacionado ao estado confusional agudo é o diagnóstico prévio de demência, estando associados, também, múltiplas comorbidades, polifarmácia - principalmente o uso de medicações de propriedades anticolinérgicas - e menor funcionalidade prévia. ${ }^{2}$

Leme et al. ${ }^{3}$ destacam que o delirium está presente em cerca de $30 \%$ dos idosos internados para cirurgias ortopédicas, estando entre os maiores desencadeantes os distúrbios hidroeletrolíticos, a dor, as infecções e dispositivos como sonda vesical de demora.

Fratura de fêmur é uma causa importante de mortalidade em idosos, estando ligada tanto a fatores pré-operatórios, como idade avançada, menor funcionalidade e síndrome demencial prévia, como variáveis pós-procedimento, incluindo tromboembolismo pulmonar, choque - principalmente cardiogênico e hipovolêmico - e infecções. ${ }^{4}$

Diante do exposto, o objetivo deste estudo foi identificar as características clínicas e funcionais dos idosos internados por fratura de fêmur e correlacioná-las com a incidência de delirium e mortalidade durante o período de internação.

\section{MÉTODOS}

Trata-se de um estudo transversal, cuja população foi composta de 90 pacientes com idade acima de 65 anos, que estiveram internados na Enfermaria de Ortopedia do Instituto de Assistência Médica ao Servidor Público Estadual (IAMSPE) por fratura de fêmur e que foram acompanhados de forma conjunta pelo Serviço de Geriatria do hospital. Os dados foram colhidos entre julho de 2017 e outubro de 2018. Todos os pacientes que aceitaram participar do estudo assinaram o Termo de Consentimento Livre e Esclarecido (TCLE). Após a assinatura do termo, os dados obtidos eram arquivados em uma planilha e, posteriormente, por meio do uso dos prontuários pessoais de cada paciente, foi realizada a correlação entre o perfil do paciente, suas intercorrências durante a internação e o desfecho.

As variáveis analisadas foram idade (em anos completos), sexo (feminino/masculino), funcionalidade (escala de Katz e Pfeffer), cronicidade de quedas (ao menos três quedas em um ano ou duas quedas em seis meses), comorbidades (diabetes mellitus/hipertensão arterial/síndrome demencial/doença pulmonar obstrutiva crônica/insuficiência cardíaca/acidente vascular cerebral/fibrilação atrial/coronariopatia/insuficiência renal crônica/neoplasia), tempo de internação (em dias completos) e medicamentos utilizados (psicotrópicos, analgésicos laxativos, protetor gástrico e anticoagulante), sendo essas variáveis correlacionadas estatisticamente com a incidência de delirium, identificado por meio do questionário Confusion Assessment Method (CAM) — realizado de forma sistemática em todos os pacientes acompanhados pelo Serviço de Geriatria do hospital e com o desfecho de mortalidade.

O projeto foi aprovado pelo Comitê de Ética e Pesquisa do Instituto de Assistência Médica ao Servidor Público Estadual de São Paulo (CEP-IAMSPE), sob o parecer no 3.037.920 e o Certificado de Apresentação para Apreciação Ética (CAAE) $n^{\circ}$ 01677518.0.0000.5463, no dia 26 de novembro de 2018 .

As análises estatísticas foram realizadas por meio do programa Statistical Package for the Social Sciences (SPSS) em sua 25a versão (2017) para Windows. ${ }^{5}$ Para tanto, fez-se uma análise univariada na qual se utilizaram os seguintes testes estatísticos: teste $t$ de Student, para amostras independentes, e teste do $\chi^{2}$. Foi considerado estatisticamente significativo o valor $\mathrm{p}<0,05$ para todos os testes.

\section{RESULTADOS}

Dos 90 pacientes selecionados, $77,8 \%(\mathrm{n}=70)$ eram mulheres; a média de idade foi de 83 anos, variando entre 65 e 99 anos; e 23,3\% ( $n=21)$ foram considerados caidores crônicos. O tempo médio de internação foi de 19,7 dias; a taxa de mortalidade geral foi de $17,8 \%(n=16)$; e foi identificado delirium em 39,9\% $(n=35)$ dos casos. A pontuação média na escala de atividades instrumentais de Pfeffer foi de 10,5 pontos, e no escore para as atividades básicas de Katz, 4,8 pontos.

A Tabela 1 mostra a correlação da presença de delirium junto aos demais fatores de risco, tendo sido estatisticamente significativa $(\mathrm{p}<0,05)$ a associação a idades mais elevadas $(\mathrm{RR}=3,0 ; \mathrm{IC} 95 \% 0,05-6,02 ; \mathrm{p}=0,046)$, menor funcionalidade tanto por baixo escore de Katz $(\mathrm{RR}=-1,0 ; \mathrm{IC} 95 \%$ $-1,80-0,24 ; p=0,011)$ quanto por elevada pontuação no Pfeffer $(\mathrm{RR}=6,7 ; \mathrm{IC} 95 \% 2,43-11,03 ; \mathrm{p}=0,002)$, uso de psicotrópicos (RR = 6,2; IC95\% 2,28-16,92; $<<0,001)$, uso de quetiapina $(R R=10,24$; IC95\% 2,65-39,53; $p<0,001)$, diabetes mellitus ( $R R=0,3 ;$ IC95\% 0,10-0,94; $p=0,034)$, síndrome demencial $(\mathrm{RR}=3,8 ; \mathrm{IC} 95 \% 1,44-10,20 ; \mathrm{p}=0,006)$ e maior desfecho de óbitos (RR = 6,6; IC95\% 1,93-22,85; $<$ < 0,001).

Óbito - demonstrado na Tabela 2 - esteve significativamente associado à presença de síndrome demencial (RR 3,6; IC95\% $1,17-11,17 ; p=0,020)$; à insuficiência renal crônica (RR 6,2; $\mathrm{IC} 95 \%$ 1,55-25,26; $\mathrm{p}=0,005)$; e à pior funcionalidade, segundo o escore de Pfeffer (RR 6,9; IC95\% 1,31-12,49; p = 0,016). 


\section{DISCUSSÃO}

Nosso estudo, realizado com 90 idosos de uma enfermaria de ortopedia, identificou que o delirium foi associado a idades mais elevadas, à menor funcionalidade, ao diabetes mellitus, à síndrome demencial, ao maior desfecho de óbitos e ao uso de psicotrópicos, especialmente a quetiapina. A mortalidade, por sua vez, foi associada à síndrome demencial, à insuficiência renal crônica e à pior funcionalidade.

Meagher et al., ${ }^{6}$ assim como nosso trabalho, também encontraram associação positiva entre síndrome demencial e delirium, o que reforça o fato de que o déficit cognitivo prévio é fator de risco importante para o desenvolvimento de estado confusional agudo. Em estudo holandês, realizado por Mosk et al., ${ }^{7}$ com idosos internados por fratura de fêmur, não foi encontrada relação entre delirium e outras comorbidades além de déficit cognitivo.

Vidal et al., em 2016, encontraram associação positiva no uso de psicotrópicos em idosos e maior incidência de delirium, informação ratificada pelos achados desta pesquisa, indicando que o uso muitas vezes inadequado de medicações, associado

Tabela 1 Características da amostra $(n=90)$ associadas ao delirium em idosos vítimas de fratura de fêmur.

\begin{tabular}{|c|c|c|c|c|}
\hline Variáveis & $\begin{array}{l}\text { Presença de delirium } \\
\qquad(\mathrm{n}=35,38,9 \%)\end{array}$ & $\begin{array}{l}\text { Ausência de delirium } \\
\qquad(\mathrm{n}=55,61,1 \%)\end{array}$ & Razão de chances & p (significância)* \\
\hline Sexo feminino & $28(40 \%)$ & $42(60 \%)$ & $0,808(0,287-2,276)$ & $0,686^{\#}$ \\
\hline Óbitos & $12(75 \%)$ & $4(25 \%)$ & $6,652(1,937-22,851)$ & $<0,001^{* *}$ \\
\hline Caidores crônicos & $10(47,6 \%)$ & $11(52,4 \%)$ & $1,600(0,596-4,293)$ & $0,349^{* *}$ \\
\hline Diabetes mellitus & $5(20,8 \%)$ & $19(79,2 \%)$ & $0,316(0,105-0,947)$ & $0,034^{* *}$ \\
\hline Hipertensão arterial & $19(36,5 \%)$ & $33(63,5 \%)$ & $0,792(0,336-1,864)$ & $0,593^{\#}$ \\
\hline Síndrome demencial & $15(62,5 \%)$ & $9(37,5 \%)$ & $3,833(1,440-10,204)$ & $0,006^{* *}$ \\
\hline DPOC & $3(60 \%)$ & $2(40 \%)$ & $2,484(0,394-15,678)$ & $0,319^{* *}$ \\
\hline Insuficiência cardíaca & $5(41,7 \%)$ & $7(58,3 \%)$ & $1,143(0,332-3,929)$ & $0,832^{* *}$ \\
\hline AVC & $4(33,3 \%)$ & $8(66,7 \%)$ & $0,758(0,210-2,735)$ & $0,672^{* *}$ \\
\hline Fibrilação atrial & $3(27,3 \%)$ & $8(72,7 \%)$ & $0,551(0,136-2,235)$ & $0,399^{* *}$ \\
\hline Coronariopatia & $1(25 \%)$ & $3(75 \%)$ & $0,510(0,051-5,106)$ & $0,560^{* *}$ \\
\hline IRC & $4(40 \%)$ & $6(60 \%)$ & $1,054(0,275-4,035)$ & $0,939^{* *}$ \\
\hline Neoplasia & $4(40 \%)$ & $6(60 \%)$ & $1,054(0,275-4,035)$ & $0,939^{* *}$ \\
\hline Psicotrópicos & $18(69,2 \%)$ & $8(30,8 \%)$ & $6,221(2,287-16,920)$ & $<0,001^{* *}$ \\
\hline Quetiapina & $13(81,3 \%)$ & $3(18,8 \%)$ & $10,242(2,653-39,538)$ & $<0,001^{* *}$ \\
\hline Haloperidol & $2(100 \%)$ & 0 & - & $0,073^{* *}$ \\
\hline Clonazepam & $4(40 \%)$ & $6(60 \%)$ & $1,054(0,275-4,035)$ & $0,939^{* *}$ \\
\hline Analgésicos & $30(38 \%)$ & $49(62 \%)$ & $0,735(0,206-2,618)$ & $0,634^{\#}$ \\
\hline Dipirona & $26(37,1 \%)$ & $44(62,9 \%)$ & $0,722(0,264-1,974)$ & $0,525^{\#}$ \\
\hline Tramadol & $18(37,5 \%)$ & $30(62,5 \%)$ & $0,882(0,377-2,063)$ & $0,773^{\#}$ \\
\hline Morfina & $6(66,7 \%)$ & $3(33,3 \%)$ & $3,586(0,834-15,418)$ & $0,072^{* *}$ \\
\hline Metadona & $3(30 \%)$ & $7(70 \%)$ & $0,643(0,155-2,672)$ & $0,541^{* *}$ \\
\hline HBM & $24(33,8 \%)$ & $47(66,2 \%)$ & $0,371(0,132-1,045)$ & $0,056^{\#}$ \\
\hline HNF & $8(57,1 \%)$ & $6(42,9 \%)$ & $2,420(0,760-7,704)$ & $0,127^{* *}$ \\
\hline
\end{tabular}

DPOC: doença pulmonar obstrutiva crônica; AVC: acidente vascular cerebral; IRC: insuficiência renal crônica; HBM: heparina de baixo peso molecular; HNF: heparina não fracionada; *valor de significância < 0,05; **teste exato de Fisher; "teste do $\chi^{2}$. 
à fragilidade inerente ao processo de envelhecimento, é fator de risco importante para os quadros confusionais. ${ }^{8}$ De modo geral, não é procedimento cirúrgico em si que é causa de confusão, mas possui contribuição importante de fatores clínicos, hemodinâmicos e farmacológicos.

Foi estatisticamente significativa a associação de delirium e mortalidade, achados compatíveis com pesquisa também realizada em outro hospital na cidade de São Paulo. Tais dados reforçam o caráter de gravidade dessa síndrome confusional aguda e considerável probabilidade de desfecho desfavorável, o que justifica uma abordagem no intuito de prevenção, identificação precoce, correção de fatores causais e manejo adequado. ${ }^{9}$

A menor funcionalidade também está associada à maior incidência de delirium, fato observado pelo nosso estudo e também por Mosk et al., ${ }^{7}$ e está intimamente relacionada ao quadro demencial, que já é identificado como variável de risco. A idade elevada, apesar de ser consagradamente correlacionada aos estados confusionais agudos, não teve significância estatística, principalmente pelo fato de o estudo só abordar idosos e as médias de idade dos dois grupos serem semelhantes.

Tabela 2 Características da amostra $(n=90)$ associadas à mortalidade em idosos vítimas de fratura de fêmur.

\begin{tabular}{|c|c|c|c|c|}
\hline Variáveis & $\begin{array}{c}\text { Óbitos } \\
\text { (n= } 16 ; 17,8 \%)\end{array}$ & $\begin{array}{l}\text { Alta hospitalar } \\
\text { (n=74, } 82,2 \%)\end{array}$ & Razão de chances & p (significância)* \\
\hline Sexo feminino & $11(15,7 \%)$ & $59(84,3 \%)$ & $1,788(0,539-5,933)$ & $0,338^{\#}$ \\
\hline Caidores crônicos & $5(23,8 \%)$ & $16(76,2 \%)$ & $1,648(0,500-5,434)$ & $0,409^{* *}$ \\
\hline Diabetes mellitus & $4(16,7 \%)$ & $20(83,3 \%)$ & $0,900(0,260-3,118)$ & $0,868^{* *}$ \\
\hline Hipertensão arterial & $12(23,1 \%)$ & $40(76,9 \%)$ & $2,550(0,752-8,641)$ & $0,124^{* *}$ \\
\hline Síndrome demencial & $8(33,3 \%)$ & $16(66,7 \%)$ & $3,625(1,176-11,171)$ & $0,020^{* *}$ \\
\hline DPOC & $1(20 \%)$ & $4(80 \%)$ & $1,167(0,122-11,193)$ & $0,894^{* *}$ \\
\hline Insuficiência cardíaca & $3(25 \%)$ & 9 (75\%) & $1,667(0,396-7,006)$ & $0,482^{* *}$ \\
\hline AVC & $3(25 \%)$ & 9 (75\%) & $1,667(0,396-7,006)$ & $0,482^{* *}$ \\
\hline Fibrilação atrial & - & $11(100 \%)$ & - & $0,100^{* *}$ \\
\hline Coronariopatia & $1(25 \%)$ & $3(75 \%)$ & $1,578(0,153-16,228)$ & $0,699^{* *}$ \\
\hline IRC & $5(50 \%)$ & $5(50 \%)$ & $6,237(1,557-25,269)$ & $0,005^{* *}$ \\
\hline Neoplasia & $1(10 \%)$ & 9 (90\%) & $0,481(0,57-4,096)$ & $0,495^{* *}$ \\
\hline Psicotrópicos & $6(23,1 \%)$ & $20(76,7 \%)$ & $1,620(0,521-5,039)$ & $0,402^{* *}$ \\
\hline Quetiapina & $4(25 \%)$ & $12(75 \%)$ & $1,722(0,474-6,253)$ & $0,405^{* *}$ \\
\hline Haloperidol & $1(50 \%)$ & $1(50 \%)$ & $4,687(0,288-82,217)$ & $0,228^{* *}$ \\
\hline Clonazepam & $1(10 \%)$ & 9 (90\%) & $0,481(0,57-4,096)$ & $0,495^{* *}$ \\
\hline Analgésicos & $11(13,9 \%)$ & $68(86,1 \%)$ & $0,194(0,050-0,747)$ & $0,010^{\#}$ \\
\hline Dipirona & $11(15,7 \%)$ & $59(84,3 \%)$ & $0,559(0,169-1,856)$ & $0,338^{\#}$ \\
\hline Tramadol & $7(14,6 \%)$ & $41(85,4 \%)$ & $0,626(0,211-1,860)$ & $0,397^{\#}$ \\
\hline Morfina & $3(33,3 \%)$ & $6(66,7 \%)$ & $2,615(0,579-11,810)$ & $0,198^{* *}$ \\
\hline Metadona & $2(20 \%)$ & $8(80 \%)$ & $1,179(0,226-6,157)$ & $0,845^{* *}$ \\
\hline HBM & $9(12,7 \%)$ & $62(87,3 \%)$ & $0,249(0,078-0,798)$ & $0,014^{\#}$ \\
\hline HNF & $5(35,7 \%)$ & $9(64,3 \%)$ & $3,283(0,925-11,646)$ & $0,056^{* *}$ \\
\hline
\end{tabular}

DPOC: doença pulmonar obstrutiva crônica; AVC: acidente vascular cerebral; IRC: insuficiência renal crônica; HBM: heparina de baixo peso molecular; HNF: heparina não fracionada; "valor de significância < 0,05; **teste exato de Fisher; "teste do $\chi^{2}$ para todas as variáveis. 
O diagnóstico prévio de diabetes mellitus foi associado, de forma estatisticamente significativa, ao delirium, o que não é habitualmente ligado a estados confusionais, mas corrobora o estudo realizado por Bisschop et al., ${ }^{10}$ que também identificaram a correlação. $\mathrm{O}$ autor explica que os níveis de cortisol, que habitualmente aumentam os níveis de glicemia, estão relacionados ao aumento de delirium, associação esta também positiva com Síndrome de Cushing, que é caracterizada por taxas de cortisol elevadas.

Assim como no delirium, o quadro demencial também implicou em uma maior taxa de óbitos, porém encontramos associação com insuficiência renal crônica, achado este não presente na maioria dos estudos de fratura de fêmur em idosos. Porém, Kim et al. ${ }^{11}$ realizaram análise de pacientes portadores de doença renal crônica não dialíticos vítimas de fratura de fêmur, e encontraram associação positiva com mortalidade intra-hospitalar, evidenciando o fato de que, apesar de tal fator de risco não ser tão difundido, também merece ser avaliado e abordado de forma adequada.

Em metanálise realizada por $\mathrm{Hu}$ et al., ${ }^{12}$ foi identificada, assim como em nossos achados, associação significativa entre a menor funcionalidade e a maior taxa de mortalidade. Os autores revelam, nesses achados, 12 preditores de mortalidade de forte evidência: idade avançada; gênero masculino; institucionalização prévia; deficiência de mobilidade; dependência para atividades de vida diária; maior classificação no escore pré-operatório de American Society of Anesthesiologists (ASA); déficit cognitivo prévio; múltiplas comorbidades; saúde mental ruim; diabetes — que, em nosso estudo, foi relacionada positivamente apenas com delirium - ; câncer; e cardiopatias prévias.

Este estudo apresenta limitações. O modelo transversal não permite realizar a definição de causalidade entre as variáveis; a amostra por conveniência pode ter selecionado um grupo homogêneo e que, eventualmente, não corresponde à realidade de outros serviços do território nacional. Além disso, o tamanho reduzido da amostra não permitiu que variáveis notadamente já consagradas pudessem ter associação estatisticamente significativa com delirium e mortalidade.

\section{CONCLUSÃO}

Este estudo identificou que em pacientes submetidos à cirurgia ortopédica, a frequência de delirium esteve associada a idades mais elevadas, à menor funcionalidade, ao diabetes mellitus, à síndrome demencial, ao maior desfecho de óbitos e ao uso de psicotrópicos; e a mortalidade, à síndrome demencial, à insuficiência renal crônica e à pior funcionalidade.

\section{AGRADECIMENTOS}

Os autores gostariam de agradecer à equipe e ao serviço de geriatria do Instituto de Assistência Médica ao Servidor Público Estadual (IAMSPE) e ao serviço de ortopedia do hospital, que gentilmente autorizaram a realização deste estudo.

\section{CONFLITO DE INTERESSES}

Os autores declaram não haver conflito de interesses.

\section{REFERÊNCIAS}

1. Bellelli G, Mazzola P, Morandi A, Bruni A, Carnevali L, Corsi M, et al. Duration of Postoperative Delirium Is an Independent Predictor of 6-Month Mortality in Older Adults After Hip Fracture. J Am Geriatr Soc. 2014;62(7):1335-40. https://doi.org/10.1111/jgs.12885

2. Oh ES, Li M, Fafowora TM, Inouye SK, Chen CH, Rosman LM, et al. Preoperative risk factors for postoperative delirium following hip fracture repair: a systematic review. Int J Geriatr Psychiatry. 2015;30(9):90010. https://doi.org/10.1002/gps.4233

3. Leme LEG, Sitta MC, Toledo M, Henriques SS. Cirurgia ortopédica em idosos: aspectos clínicos. Rev Bras Ortop. 2011;46(3):238-46. http:// dx.doi.org/10.1590/S0102-36162011000300002

4. Smith T, Pelpola K, Ball M, Ong A, Myint PK. Pre-operative indicators for mortality following hip fracture surgery: a systematic review and meta-analysis. Age Ageing. 2014;43(4):464-71. https://doi.org/10.1093/ ageing/afu065

5. IBM. SPSS Statistics Version 25.0. Estados Unidos: IBM; 2017.

6. Meagher D, O'Regan N, Ryan D, Connolly W, Boland E, O'Caoimhe R, et al. Frequency of delirium and subsyndromal delirium in an adult acute hospital population. Br J Psych. 2014;205(6):478-85. https:// doi.org/10.1192/bjp.bp.113.139865
7. Mosk CA, Mus M, Vroemen JP, van der Ploeg T, Vos DI, Elmans LH, et al. Dementia and delirium, the outcomes in elderly hip fracture patients. Clin Interv Aging. 2017;12:421-30. https://doi.org/10.2147/CIA.S115945

8. Vidal X, Agustí A, Vallano A, Formiga F, Moyano AF, García J, et al. Elderly patients treated with psychotropic medicines admitted to hospital: associated characteristics and inappropriate use. Eur J Clin Pharmacol. 2016;72(6):755-64. https://doi.org/10.1007/s00228-016-2032-2

9. Silva TJA. Delirium em idosos hospitalizados: análise de características clínicas e prognóstico [dissertação]. São Paulo: Universidade de São Paulo; 2015.

10. Bisschop PH, de Rooij SE, Zwinderman AH, van Oosten HE, van Munster BC. Cortisol, Insulin, and Glucose and the Risk of Delirium in Olde Adults with Hip Fracture. J Am Geriatr Soc. 2011;59(9):1692-6. https:// doi.org/10.1111/j.1532-5415.2011.03575.x

11. Kim SM, Long J, Montez-Rath M, Leonard M, Chertow GM. Hip Fracture in Patients With Non-Dialysis-Requiring Chronic Kidney Disease. J Bone Miner Res. 2016;31(10):1803-9. https://doi.org/10.1002/jbmr.2862

12. Hu F, Jiang C, Shen J, Tang P, Wang Y. Preoperative predictors for mortality following hip fracture surgery: A systematic review and meta-analysis. Injury. 2012;43(6):676-85. https://doi.org/10.1016/j.injury.2011.05.017 